\title{
Analysis of attribute domain for geometrical gesture performed by arm movements
}

\author{
Wan Khairunizam ${ }^{1}$, Khairul Ikram ${ }^{2}$, Hafiz Halin ${ }^{3}$, Azri Aziz ${ }^{4}$, I. Zunaidi ${ }^{5}$, S. A Bakar ${ }^{6}$, \\ Z. M Razlan ${ }^{7}$, W. A. W. Mustafa ${ }^{8}$ \\ ${ }^{1,2,3,6,7}$ School of Mechatronic, University Malaysia Perlis Pauh Putra Campus, Malaysia \\ ${ }^{4,8}$ Faculty of Engineering Technology, University Malaysia Perlis, Malaysia \\ ${ }^{5}$ Sustainable Advanced Manufacturing (SAM), The Industrial Center, University Sunderland, United Kingdom
}

\begin{abstract}
Article Info
Article history:

Received Jan 19, 2019

Revised Apr 17, 2019

Accepted May 11, 2019

\section{Keywords:}

Arm trajectory

Attribute

Gesture recognition

Ontology

ABSTRACT

Recently, the ability of the recognition systems to recognize gestures produced by human increases because of the advancement of the decisionmaking algorithm or the classifier. However sometimes the systems miss recognize the unknown gesture because the decision-making algorithm is in chaotic. The situation happens when the unknown gesture brings high similarity values compared with gestures stored in the database. Therefore, by adding a knowledge context of the gesture could reduce the chaotic and the systems output precise decision. The study proposes to employ the concept of ontology in the recognition of the arm gesture; however, this paper discusses the development of the attribute domain of the arm gesture. The attribute domain is defined as a stage where all the characteristic arm movement are presented. The characteristic arm movements are the features of the geometrical gesture. All the features are stored as the elements of the attribute domain. The relations among the elements must be formed and analyzed. Two analyses are conducted, which are the statistical and the precision measures. The statistical results have statistically significant to classify 9 registered gesture. Moreover, the precision measure is successfully removing the common data for all 9 gestures.
\end{abstract}

Copyright @ 2019 Institute of Advanced Engineering and Science. All rights reserved.

Corresponding Author:

Wan Khairunizam Wan Ahmad, School of Mechatronic, University Malaysia Perlis Pauh Putra Campus, 01000 Arau, Perlis, Malaysia .

Email: khairunizam@unimap.edu.my

\section{INTRODUCTION}

Human-Computer Interaction (HCI) becomes one of the communication tools in the era of industrial revolution 4.0. It is evolving from the cursor applications on desktop computers to the advancement of new technology. The modern HCI application utilizes human motion, such as gait pattern, facial expression and gesture. In the future, smartphone interaction, television manipulation, games control, and electric vehicles manipulation will use body gestures as the medium for the interaction.

Latest research reported, one of the latest technologies utilized by the researchers to interact with the human gesture is Kinect sensor $[1-3,21]$. The ability of the Kinect systems to recognize and track dynamic movement of human body is suitable for gaming and medical sciences applications.

Previously, most of the researchers focus on investigating various type of sensors to record the upper body movement including the arm. The various features from arm movements are extracted and investigated to increase the accuracy of the decision-making systems to recognize human gesture [4-9].

The study proposes to employ the concept of ontology in the recognition of the arm gesture; however, this paper discusses the development of the attribute domain of the arm gesture. The attribute 
domain is defined as a stage where all the characteristic arm movement are presented [10]. The characteristic arm movements are the features of the geometrical gesture. Qualisys Track Manager System (QTM) is used in the experiments. Ten (10) subjects are chosen to perform the gestures with the repetition of 10 trials for each. One (1) marker is attached to the right hand and the subject is asked to perform the gesture. QTM produces three-dimensional coordinate data in $x$-, $y$ - and $z$ - axes of the moving marker. The acquired 3D data are used to analyze the attribute domain of geometrical gesture.

This paper is organized as follows: Section 2 discusses the related works of the research. Section 3 describes the proposed research methodologies. Section 4 discusses the finding of the research and the conclusions of the research works are discussed in section 5.

\section{RELATED WORKS}

Ontology system can be applied in various field of study. In 2013, H. Fujita develop a medical databased called as a reference ontology [14]. A subjective and objective attribute alignment is proposed and employed to create medical diagnosis systems. The alignment is pondered from medical ontology to rank the attribute for a decision making by using fuzzy reasoning. The intelligent reasoning system is used to develop Virtual Doctor System (VDR). In the meantime, S. Santos develops a system named Support System for Brain Tumor's Therapeutic Planning (SATTC) by using of elicit attribute of brain tumor database to provide a complete reference system for a doctor, especially neurologist, neurosurgeon, radiologist and pathologist in order to decide the best treatment plan for the patient [15].

Apart from a medical science, the application of ontology also adopted in e-commerce systems. In 2015, A. Horch by using product attributes to supply a comparison between all the prices in the online market [16]. This system can help online shoppers get the best price and as a reference to the online retailer to offer the best price to compete with another retailer.

In the research of pattern recognition, there are several researchers conduct investigation on object's shape by understanding the attributes of the object such as overall shape, holes, curvature, sharpness and smoothness of the object or sculpture [17-18]. F. Fouhey defines a set of 3D shape attributes included planarity, symmetry and occupied space to deduce all the common 3D shape properties by the exterior appearance. R. Brath uses 3D object attribute to create guidance for information visualization in art [17]. Some of the attribute terminologies used are termination, closure, hole, curvature, added marks, angle and intersection.

In ontology, attribute is defined as everything that exist and represent every concept that connected by sharing similar characteristic. The connection between these concepts is used to retrieve data and information $[11-13,22]$. This paper discusses the development of the attribute domain of geometrical shape gesture. The information obtained from the literature review are referred to develop the attribute domain and is discussed in detail in chapter 3.

\section{RESEARCH METHODOLOGIES}

The flow of the research is shown in Figure 1. A set of geometrical shape gesture is used in the experiments. The attribute domain of ontology is designed by using data produced from the geometrical shape gesture. Geometrical shape as shown in Figure 2.

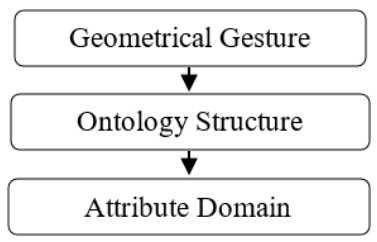

Figure 1. Flow of the research

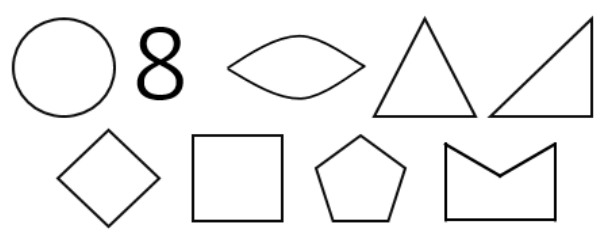

Figure 2. Geometrical shape

\subsection{Geometrical Shape Gesture}

Nine (9) geometrical shapes circle, number 8, eye, triangle, right triangle, diamond, rectangle, polygon and irregular polygon were selected to be performed by the subjects. The geometrical shapes gesture is shown in Figure 2 and is presented in (1). Qualisys Track Manager System (QTM) [19] was used in the experiments. Ten (10) subjects were chosen to perform the gestures with the repetition of 10 trials for each. 
One marker was attached to the right hand and the subject was asked to perform the gesture. QTM produces three-dimensional coordinate data in $x$-, $y$ - and $z$ - axes of the moving marker.

$\mathbf{G}=\{$ circle, number 8, eye, triangle, right triangle, diamond, rectangle, polygon and irregular polygon $\}$

\subsection{An Ontological Structure of Geometrical Shape Gesture Performer by the Right Hand}

In this research, by referring to Figure 3, an ontological structure is divided to three stages, which are knowledge, attribute and process domains [10]. All the processes until $\boldsymbol{D}_{\boldsymbol{G}}$ was produced in a knowledge domain as shown in Figure 4.

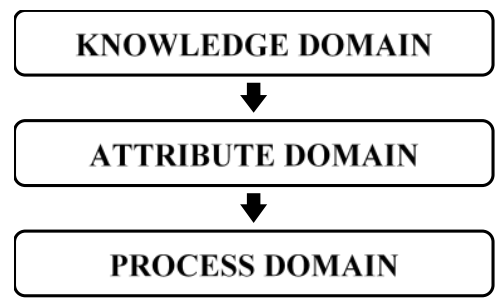

Figure 3. Three domains in the ontology approach [20]

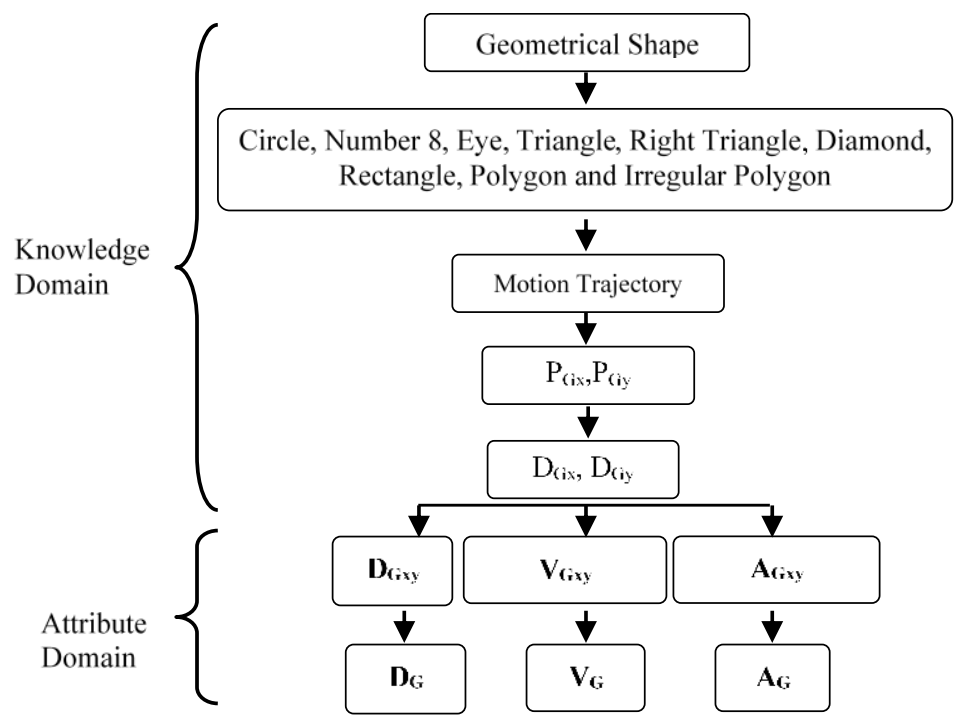

Figure 4. Presentation of knowledge and attribute domains

QTM produces 3D data of hand movements or the 3D position data of the moving marker $(x, y, z)$. In the preliminary experiments, the significant of 3D data was investigated. The investigation results showed the depth data or $\mathrm{z}$-axis was not significant and it was removed.

The collected raw data from QTM were defined as position, $\boldsymbol{P}_{G}=\left(P_{G x}, P_{G y}\right)$. The position data were processed by using resampling algorithm to produce distance, $\boldsymbol{D}_{G}=\left(D_{G x}, D_{G y}\right)$ [20]. $\boldsymbol{G}$ is the registered gesture in the experiments.

\subsection{Attribute Domain of Geometrical Shape Gesture, $G$}

Distance data $\boldsymbol{G}$ from the knowledge domain were further processed in the attribute domain. The attribute domain has data called element or attribute element. The elements of the attribute domain were velocity $\boldsymbol{V}_{G}=\left(V_{G x}, V_{G y}\right)$ and acceleration $\boldsymbol{A}_{G}=\left(A_{G x}, A_{G y}\right)$ as shown in (2) and (3), respectively.

$$
\begin{aligned}
& \operatorname{VG}(n)=\{D G(n+1)-D G(n)\} / t \\
& A G(n)=\{V G(n+1)-V G(n)\} / t
\end{aligned}
$$


Where,

$n=$ number of data.

$t=$ time interval between two data.

$G=$ the registered gesture in the experiments.

Moreover, $x$ - and $y$-axes data were combined by using mathematical relation to form $D_{G x y}, V_{G x y}$, and $A_{G x y}$ as shown in (4), (5), and (6), respectively to increase data diversity. $D_{G x y}, V_{G x y}$ and $A_{G x y}$ are the movement of $\boldsymbol{D}_{G}$, the velocity $\boldsymbol{V}_{\boldsymbol{G}}$ and the acceleration $\boldsymbol{A}_{\boldsymbol{G}}$ of the hand as shown in Figure 4.

$$
\begin{aligned}
& D G=\text { DGxy }=\sqrt{ }(\text { DGx } 2+\text { DGy } 2) \\
& V G=V G x y=\sqrt{ }(\text { VGx } 2+\text { VGy } 2) \\
& A G=A G x y=\sqrt{ }(\text { AGx } 2+\text { AGy } 2)
\end{aligned}
$$

\subsection{Investigation of Attribute Element}

The attribute elements were built from 9 geometrical shape gestures G. In the data collection experiments, 10 subjects were selected to perform the gestures, and each gesture with 10 trials or repetition. In the preprocessing, each signal was resampled to 30 points. These data built the attribute elements. Two investigation conducted, which were a statistical analysis and a precision measure.

A statistical analysis was conducted to test the redundancy of attribute element with reflect to 9 registered gesture G. To test the redundancy or the significant level of attribute elements, an ANOVA method was used, which was a parametric statistical technique used to compare datasets. The analysis of variance in ANOVA was applied to compare two or more datasets by comparing means and the relative variance. This paper uses one-way ANOVA to find the significant measure between all the geometrical shape data. From the ANOVA reports, if $\mathrm{P}$ value is $<0.05$, so the null hypothesis can be rejected and mean significantly different than another group.

A precision measure was used to investigate how close the distance between 2 set of data or dataset. The results are used investigate to group or to remove among set of data and are further explain in chapter 4.

\section{RESULTS AND ANALYSIS}

\subsection{Element of Attribute Domain}

Figures 5, 6 and 7 show the presentation of 9 geometrical shape gestures in attribute domain, which are distance $\boldsymbol{D}_{\boldsymbol{G}}$, velocity $\boldsymbol{V}_{\boldsymbol{G}}$, and acceleration $\boldsymbol{A}_{\boldsymbol{G}}$. $\boldsymbol{G}$. Each gesture consists of 30 data points resulted from the resampling algorithm. Data are rescaled to the range of $[0,1]$. The purpose of rescaling is easier for the comparison between data.

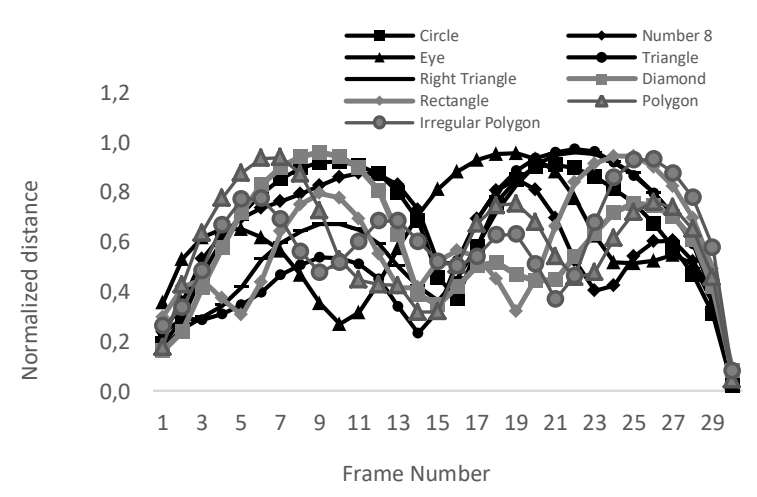

Figure 5. Presentation of Distance $\boldsymbol{D}_{G}$

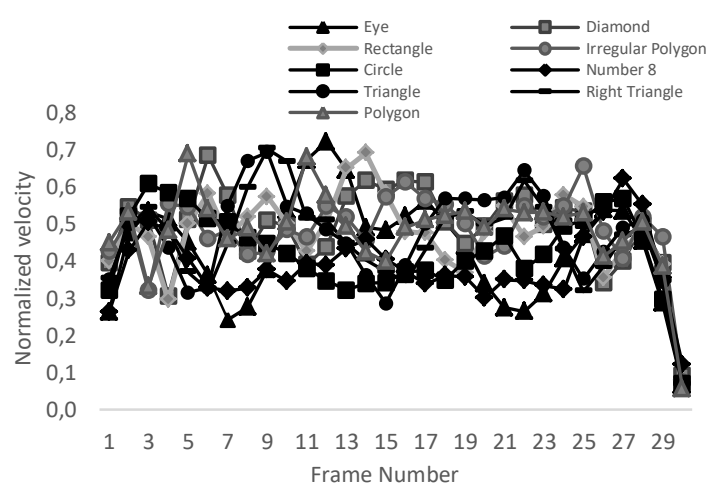

Figure 6. Presentation of Velocity $\boldsymbol{V}_{\boldsymbol{G}}$ 


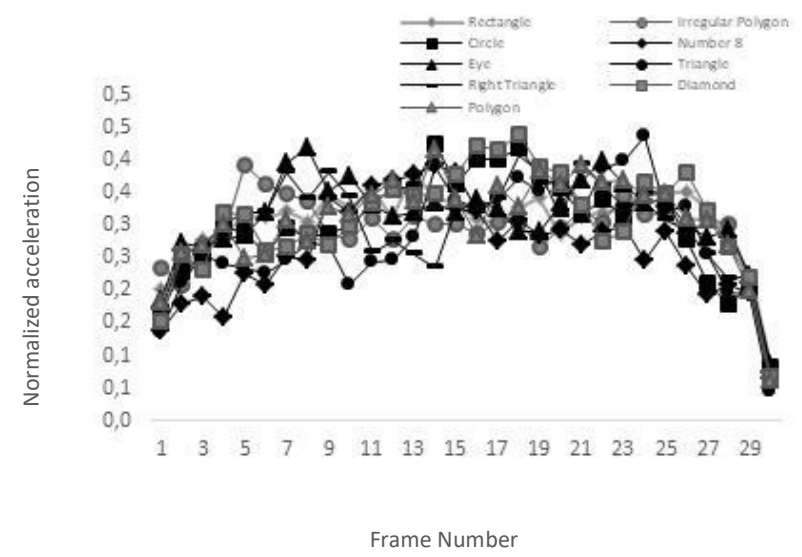

Figure 7. Presentation of Acceleration $\boldsymbol{A}_{\boldsymbol{G}}$

\subsection{Investigating Elements of Attribute Domain}

4.2.1 A Statistical Investigation

Figure 8 shows the ANOVA results for all three tested datasets and is summarized in Table 1.

Table 1. ANOVA Results

\begin{tabular}{c|ccc}
\hline Features & $p$-value & Actual $p$-value & $F$-statistic \\
\hline $\boldsymbol{D}_{\boldsymbol{G}}$ & $<0.001$ & $6.93 \times 10^{-61}$ & 38.16 \\
$\boldsymbol{V}_{\boldsymbol{G}}$ & $<0.001$ & $7.9 \times 10^{-85}$ & 52.37 \\
$\boldsymbol{A}_{\boldsymbol{G}}$ & $<0.001$ & $7.2 \times 10^{-16}$ & 11.15 \\
\hline
\end{tabular}

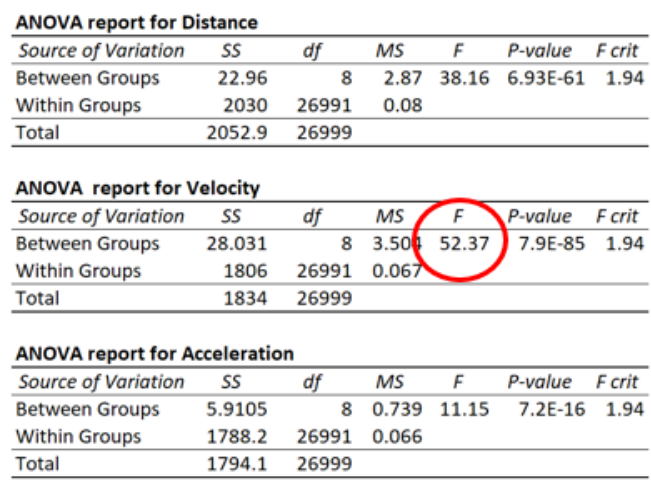

Figure 8. ANOVA results of $\boldsymbol{D}_{G}, \boldsymbol{V}_{\boldsymbol{G}}$ and $\boldsymbol{A}_{\boldsymbol{G}}$

By referring to Table $1, p$-value for $\boldsymbol{D}_{G}, \boldsymbol{V}_{\boldsymbol{G}}$ and $\boldsymbol{A}_{\boldsymbol{G}}$ are less than 0.05 , which mean that they are statistically significant between the selected 9 geometrical shape gestures. On the other hand, $F$-statistic shows the velocity dataset possess highest value followed by distance. Acceleration datasets gives the lowest $F$ value, but still significant where $F>1$.

\subsubsection{A Precision Measures Investigation}

Figures 9, 10 and 11 show the results plotted based on 3 precision measure's tools, which are the range of a maximum and minimum, a mean and a standard deviation for $\boldsymbol{D}_{\boldsymbol{G}}, \boldsymbol{V}_{\boldsymbol{G}}$ and $\boldsymbol{A}_{\boldsymbol{G}}$. Each signal represented by 30 resampled points. The points have the similar characteristic if they are placed close to each other. The round marker in Figure 9 shows that resampled point \#1 and \#30 have similar characteristic. Consequently, both points can be place in the same group. The round marker in Figure 10 shows that the resampled point \#2 and \#30 have similar characteristic. The triangle marker shows that the resampled points are place away from each other in the space. Consequently, further investigation is conducted to find out the differences between them. 


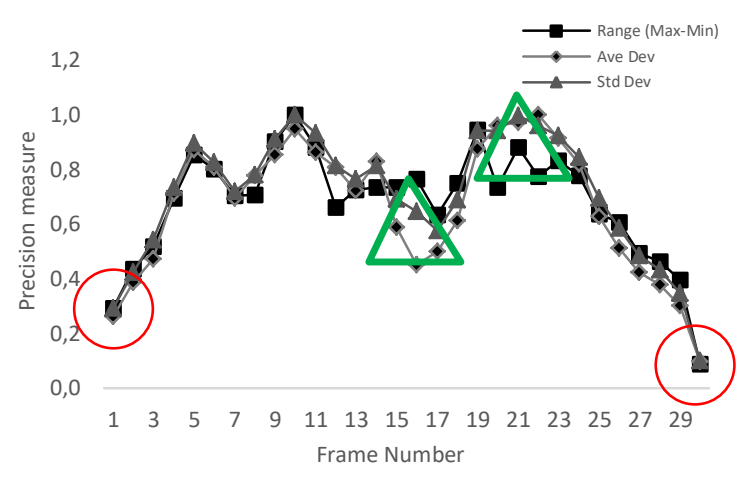

Figure 9. Precision measure for distance dataset

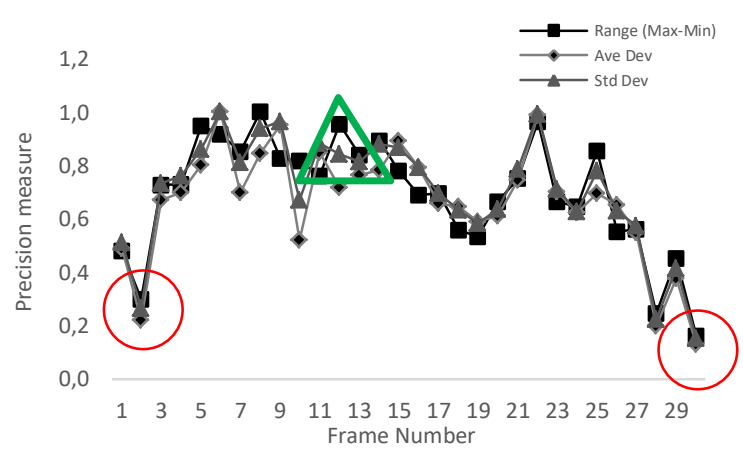

Figure 10. Precision measure for velocity dataset

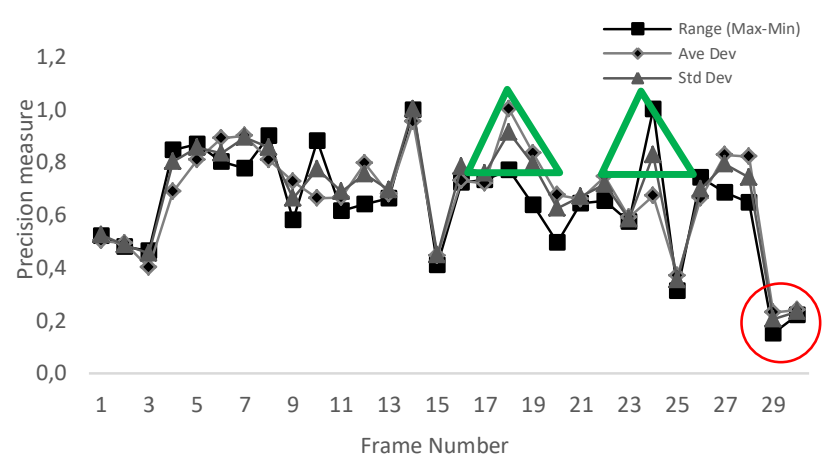

Figure 11. Precision measure for acceleration dataset

\section{CONCLUSIONS}

The aim of this paper is to propose the methods for designing the attribute domain of arm that presents geometrical shape gesture. The attribute domain is one of the elements from ontology framework. The elements of an attribute domain are defined as distance $\left(\mathbf{D}_{\mathbf{G}}\right)$, velocity $\left(\mathbf{V}_{\mathbf{G}}\right)$, and acceleration $\left(\mathbf{A}_{\mathbf{G}}\right)$ of the hand. The elements are designed by using arm movements data collected by using Qualisys Track Manager System (QTM). The data are pre-processed by using resampling and normalizing algorithms before forming the attribute elements. The relations among the elements must be formed and analyzed. Two analyses are conducted, which are the statistical and the precision measures. The results from both analyses show that the proposed methods are significant to represent knowledge of the registered gesture. In the future, the research will be continued by building the process domain where it will be combined with Fuzzy technique to recognize arm gesture.

\section{ACKNOWLEGDMENT}

The author would like to acknowledge the support from the Fundamental Research Grant Scheme (FRGS) under a grant number of FRGS/1/2017/TK04/UNIMAP/01/2 from the Ministry of Education Malaysia.

\section{REFERENCES}

[1] H. M. Jais, Z. R. Mahayuddin, and H. Arshad, "A Review on Gesture Recognition Using Kinect," 5th Int. Conf. Electr. Eng. Informatics 2015, pp. 594-599, 2015.

[2] S. J. S. A. Fathima, "Review on Microsoft Kinect' S Gesture Technology Based Robotics," 2017 International Conference on Innovations in Information, Embedded and Communication Systems (ICIIECS), 2017.

[3] J. O. Reinaldo, R. S. Maia, and A. A. Souza, "Adaptive navigation for mobile robots with object recognition and ontologies,” Proc. - 2015 Brazilian Conf. Intell. Syst. BRACIS 2015, pp. 210-215, 2016. 
[4] J. S. Sonkusare, N. B. Chopade, R. Sor, and S. L. Tade, “A Review on Hand Gesture Recognition System,” 2015 Int. Conf. Comput. Commun. Control Autom., pp. 790-794, 2015.

[5] L. Chen, F. Wang, H. Deng, and K. Ji, "A Survey on Hand Gesture Recognition”, 2013.

[6] R. R. Itkarkar and A. V. Nandi, "A survey of $2 D$ and $3 D$ imaging used in hand gesture recognition for humancomputer interaction (HCI)," WIECON-ECE 2016 - 2016 IEEE Int. WIE Conf. Electr. Comput. Eng., no. December, pp. 188-193, 2017.

[7] H. Cheng, L. Yang, and Z. Liu, "A Survey on 3D Hand Gesture Recognition," IEEE Trans. Circuits Syst. Video Technol., vol. PP, no. 99, p. 1, 2015.

[8] L. Y. Deng, D. Lee, Y. Liu, and N. C. Tang, "Human Computer Interaction Based on Hand Gesture Ontology," Gesture, pp. 26-31, 2007.

[9] Wan Khairunizam, K. Ikram, S. A. Bakar, Z. M. Razlan, I. Zunaidi, "Ontological Framework of Arm Gesture Information for the Human Upper Body," in Intelligent Manufacturing \& Mechatronics, 2018, pp. 507-515.

[10] W. Fang, Y. Guo, and W. Liao, "Ontology-based indexing method for engineering documents retrieval," 2016 IEEE Int. Conf. Knowl. Eng. Appl. ICKEA 2016, pp. 172-176, 2016.

[11] P. Gu, J. Xu, C. Li, and Y. Duan, "Towards mapping large scale ontologies based on RFCA with attribute reduction," Proc. - 2008 IFIP Int. Conf. Netw. Parallel Comput. NPC 2008, pp. 407-411, 2008.

[12] K. Chen, W. Zuo, F. Zhang, and F. He, "Multiple attribute mappings for domain ontology generation in deep web," 2010 2nd Conf. Environ. Sci. Inf. Appl. Technol. ESIAT 2010, vol. 3, pp. 215-218, 2010.

[13] J. Xu, P. Gu, C. Li, and Y. Duan, "Enhancing Rough Set and Formal Context based Ontology Mapping Method with Attribute Reduction,” 2008 Third Int. Conf. Pervasive Comput. Appl., pp. 385-388, 2008.

[14] H. Fujita, "Fuzzy reasoning for medical diagnosis based on subjective attributes and objective attributes alignment," Proc. 2013 Jt. IFSA World Congr. NAFIPS Annu. Meet. IFSA/NAFIPS 2013, pp. 950-955, 2013.

[15] R. S. Santos et al., "Using reference ontology to elicit attributes of brain tumors database," Int. Conf. Inf. Soc. iSociety 2014, pp. 181-186, 2015.

[16] A. Horch, H. Kett, and A. Weisbecker, "Extracting product unit attributes from product offers by using an ontology,” 2015 2nd Int. Conf. Comput. Sci. Comput. Eng. Soc. Media, CSCESM 2015, pp. 67-71, 2015.

[17] R. Brath, "Multiple shape attributes in information visualization: Guidance from prior art and experiments, " Proc. Int. Conf. Inf. Vis., pp. 433-438, 2010.

[18] D. F. Fouhey, A. Gupta, and A. Zisserman, "3D Shape Attributes," 2016 IEEE Conf. Comput. Vis. Pattern Recognit., pp. 1516-1524, 2016.

[19] H. D. K. Ikram, Wan Khairunizam, Azri A. Aziz, S. A. Bakar, Z. M. Razlan, I. Zunaidi, "Adaptive Gesture Recognition Based on Human Physical Characteristic," 2018 IEEE 14th Int. Colloq. Signal Process. Its Appl., pp. 129-134, 2018.

[20] K. Ikram, W. Khairunizam, and A. A. Aziz, "Ontological Approach on Designing Knowledge Domain Presentation of MOCAP based on Arm Movement," 2018 International Conference on Intelligent Informatics and Biomedical Sciences (ICIIBMS), pp. 1-6, 2018.

[21] Rosdiyana Samad, Law Wen Yan, Mahfuzah Mustafa, Nor Rul Hasma Abdullah, Dwi Pebrianti," Multiple Human Body Postures Detection using Kinect," Indonesian Journal of Electrical Engineering and Computer Science (IJEECS),Vol. 10, No. 2, pp. 528-536, 2018.

[22] Taurayi Rupere, "An Evaluation of language Ontology Web Based Assessment System," Indonesian Journal of Electrical Engineering and Computer Science (IJEECS),, Vol. 2, No. 3, pp.112-122, 2013.

\section{BIOGRAPHIES OF AUTHORS}
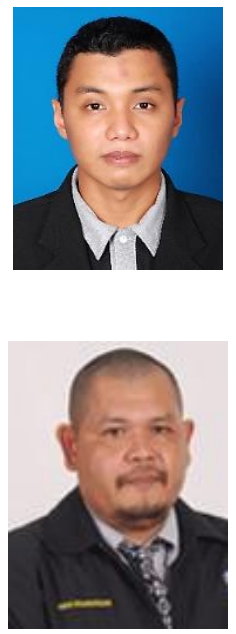

Khairul Ikram is a third-year master's degree student in Mechatronic Engineering. His main research center on development of ontology framework for arm gesture recognition. The research aims to apply the ontological concept in engineering application. He received a bachelor's degree in Industrial Electronic Engineering from University Malaysia Perlis, Malaysia in 2015. He is actively involved as a committee member of International Conference on Innovation Engineering Technology and International Conference on Mechanical Electronic and Biosystem Engineering conference series. He is interested about artificial intelligent in robotic development. His vision is to create human-friendly robot in the future.

Wan Khairunizam received his B. Eng. degree in Electrical \& Electronic Eng. from Yamaguchi University and Ph.D. in Mechatronic Eng. from Kagawa University, in 1999 and 2009, respectively. He is currently a Senior Lecturer at School of Mechatronic Engineering, University Malaysia Perlis. He is the members of Board of Engineer and Institute of Engineer, Malaysia and IEEE. His research interest is in Human-Computer Interaction (HCI), Intelligent Transportation System, Artificial Intelligence and Robotics. 


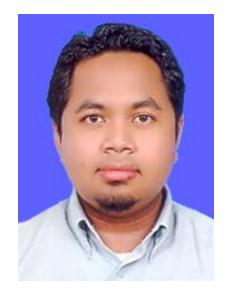

Azri A. Aziz received his B. Eng.in Mechatronic engineering from International Islamic University of Malaysia in 2009, and Master degree in Mechatronic From UniMAP in 2017. After graduating from his bachelor degree he joined Megatech International College where he worked as lecture to supervise engineering diploma student. He did his Msc studying human behavioral, gesture recognition and Human Computer Interaction. He is currently a teaching engineer in UniMAP, Perlis. His current research interest is in Artificial Intelligence, Robotic and Automation Engineering, Industrial Agriculture Automation and IOT.

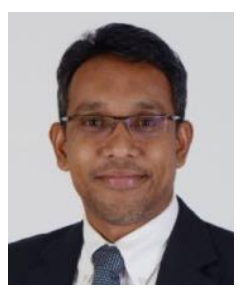

Z. Ibrahim was working as a Chief Operating Officer at Technopreneur at UNIMAP, University Malaysia Perlis, Malaysia. He has been working as Senior Lecturer at UNIMAP. He has obtained his B. Eng. in Mechanical Engineering and PhD in System Engineering (Robotic) from Mie University, Japan in 1997 and 2006 respectively. He has involved and successfully lead several research activities or project such as Mobile Robot Dead-Reckoning System development, Development of Autonomous Mapping and Navigation System, Electromyography (EMG) signals analysis and controlling prosthesis arm robot development funded by fundamental research grant and e-Science fund. Now, he is working as a Senior Research Fellow at University Sunderland, UK and doing his research in Sustainable Advanced Manufacturing.

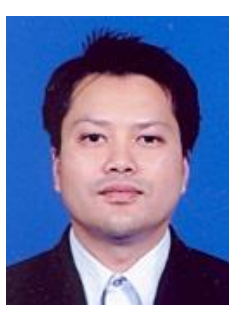

S.A Bakar received his B. Eng., M. Eng., and Ph.D. degrees in Mechanical engineering from Mie University, Mie, Japan in 1997, 2004 and 2010, respectively. After graduating from his bachelor's degree he joined NEC Semiconductors (Malaysia) where he worked as machine engineer to supervise engineering team in order to improve equipment performance and efficiency. He did his Ph.D. studying human and robot collaborative tasks in senses and ergonomics. He is currently a senior lecturer in UniMAP, Perlis. His current research interest is in Medical Engineering, Industrial Agriculture Automation and sustainable energy.

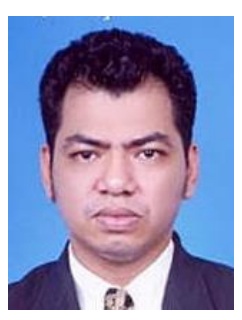

Zuradzman M. Razlan received his Bachelor of Mechanical Engineering from Yamagata University, Japan from Apr. 1993-Mar. 1997 and Ph.D. in Engineering from Mie University, Japan. He is currently Senior Lecturer at School of Mechanical Engineering, University Malaysia Perlis and experience in his field almost 11 years in R\&D Design Engineering. His research interest is in Energy, Thermo-Fluid, Two Phase Flow, Air Flow System, Heat-Pump and refrigeration system.

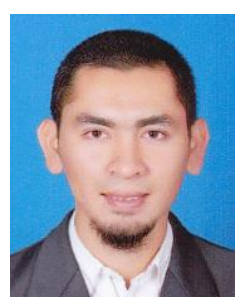

W. Azani W. Mustafa received degree in Biomedical Electronic Engineering (2013) and PhD in Mechatronic Engineering (2017) from University Malaysia Perlis (UniMAP). Registered on Board Engineer Malaysia (BEM) on 2014 and Malaysia Board of Technologists (MBOT) on 2017. Published more than 53 academic articles (Scopus H-Index 8) and 1 book. Now, working as a senior lecturer at University Malaysia Perlis, Malaysia. Current interest includes image processing, biomechanics, intelligence system, and control system.

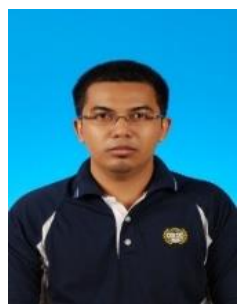

Hafiz Halin received a degree in Mechatronic Engineering (2012) from University Teknikal Malaysia Melaka (UTeM). Registered on Board Engineer Malaysia (BEM) in 2018. Published more than six academic articles (Scopus H-Index). Currently, Master of Science (MSc) student at University Malaysia Perlis, Malaysia. Research interest includes autonomous vehicle and intelligence system. 